\title{
DIFUSIÓN Y LOCALIZACIÓN INDUSTRIAL EN ESPAÑA DURANTE EL PRIMER TERCIO DEL SIGLO XX *
}

CONCHA BETRÁN PÉREZ

Universidad de Valencia

\section{RESUMEN}

El propósito del trabajo es estudiar la difusión de los cambios hacia una estructura industrial manufacturera diversificada en las diferentes regiones españolas durante el primer tercio del siglo $\mathrm{xx}$ y la evaluación de los factores que explican el crecimiento de la industria manufacturera de estas regiones. Los factores considerados son las economías de localización (externalidades marshallianas) y las economías de urbanización (externalidades de Jacobs). Como no existe un censo industrial en España, se ha estimado una reconstrucción de los impuestos directos industriales en 1913 y 1929 para 50 provincias y 20 manufacturas. En este período de creación de una importante base manufacturera para realizar el proceso de industrialización la mayoría de las regiones no incrementaron su especialización e incluso las economías de urbanización fueron positivas para el crecimiento manufacturero entre 1913 y 1929.

\section{ABSTRACT}

The aim of this work is to study the diffusion of the changes towards a diversified manufacturing structure in the different Spanish regions during the first third of the XXth century and the evaluation of the factors which explain the manufacturing industrial growth of these regions. The considerated

* Agradezco los comentarios y sugerencias de Jordi Palafox, Jose E. Boscá, Carles Sudrià, Jordi Maluquer, Sebastián Coll y dos evaluadores anónimos. Una anterior versión de este trabajo ha sido presentado al II Encuentro de Historia Económica. Universidad Menéndez Pelayo, Valencia, abril 1997, parte de él proviene de mi tesis doctoral.

Revista de Historia Económica

Año XVII, Otoño-Inviemo 1999. N."3. 
factors are the localization economies (Marshall's externalities of specialization) and the urbanization economies (externalities of diversity or Jacobs's externalities). Owing to the non-existence of an industrial census in Spain, we estimate a data set based on a reconstruction of the industrial direct taxes in 1913 and 1929, for 50 provinces and 20 manufactures. We find that in this period of creating of an important manufacturing basis to realize the industrialization process, the different regions did not increase their specialization and, the urbanization economies were even positive to the manufacture growth between 1913 and 1929.

En la economía española, durante el primer tercio del siglo xx, se produjeron importantes cambios en la participación del sector secundario ${ }^{1}$, acompañados de modificaciones relevantes en su estructura. Estas transformaciones estuvieron asociadas a destacadas alteraciones en la oferta y la demanda. A medida que un país avanza en su proceso de industrialización su tejido industrial se hace más complejo y ganan importancia los subsectores de bienes intermedios y de equipo, suministradores a su vez de bienes a otras industrias. De esta manera, en España, en el período entre 1913 y 1929, que se va a estudiar en el presente trabajo, tuvo lugar un aumento en la participación de la energía, los transformados metálicos, la química y la primera transformación de metales, y una disminución del textil y la alimentación. La demanda cada vez más estable procedente de la propia industria, en la mayoría de los casos, ocasionó su desarrollo progresivo y un mayor tamaño del sector. Al mismo tiempo, la mayor interconexión entre las industrias, a la que contribuyeron especialmente los subsectores transmisores del cambio tecnológico, dio lugar a numerosos efectos positivos ${ }^{2}$.

Una mejor comprensión de un proceso tan complejo no puede eludir el análisis regional. Por dos razones: por un lado, por la obtención de un mayor número de observaciones sobre un mismo fenómeno y, por otro, por cuanto que las relaciones económicas se llevan a cabo en el espacio, se producen en lugares concretos que son fruto de la concentración de personas, empresas, recursos naturales, factores de producción, infraes-

${ }^{1}$ A. Carreras (1984, 1987 y 1990). La participación de la producción industrial en el producto utilizando los datos procedentes de Alcaide, pasó de un 20 por 100 en 1915 a un 31 por 100 en 1930. Otro período de cambio importante en la distribución del producto fue entre 1950 y 1965, lo hizo del 31 al 39 por 100. En los datos de población activa industrial ésta asciende en el primer periodo considerado del 19 al 32 por 100 y en el segundo del 26 al 36 por 100 .

${ }^{2}$ C. Betrán (1997). 
tructuras, etc..., por lo que considerar su localización ayuda a entender el funcionamiento de la economía ${ }^{3}$. El objetivo de este trabajo es estudiar, a través de este tipo de análisis, tanto el grado de difusión de los cambios que tuvieron lugar en la industria como evaluar algunos de los factores explicativos del crecimiento industrial de las diferentes regiones.

Las fuerzas directoras de la localización de la industria en el espacio tienen el interés de mostrar: la importancia de la dotación de recursos, la demanda, el cambio y la difusión tecnológicas y la actuación de los rendimientos crecientes. Asimismo, la concentración del sector secundario en determinados centros industriales, asociados a una apreciable diversificación de su estructura productiva, permite confirmar la actuación de destacados efectos de encadenamientos de demanda hacia adelante y hacia atrás y fuertes externalidades de localización y urbanización, de relevancia indiscutible en la transformación de una economía agraria en una industrial.

De este modo, la organización del artículo se divide en los apartados siguientes: 1) una breve síntesis de algunas de las principales aportaciones teóricas sobre la localización útiles para analizar el caso español; 2) la estimación del nivel de industrialización, industria manufacturera por habitante, en las 50 provincias españolas a través del indicador de los impuestos directos industriales y sus cambios entre 1913 y 1929; 3) el estudio de la difusión de las transformaciones en la estructura industrial en las distintas provincias españolas mediante la determinación del grado de especialización y sus variaciones; 4) el análisis de la relevancia de las economías de localización y de diversificación en la trayectoria industrial provincial, y en el 5), por último, la explicación de las conclusiones más destacadas.

\section{LOCALIZACIÓN INDUSTRIAL}

Las teorías tradicionales sobre la localización industrial se han basado en gran medida en los aspectos relacionados con los costes de transporte. Éstos condicionan las decisiones de ubicación al ser parte importante del total de los costes de producción, y más todavía en el pasado, interviniendo en el suministro de las materias primas y en la distribución de los productos. Entre las aproximaciones más relevantes sobresalen las aportaciones de Weber ${ }^{4}$, fundadas en la variación de los costes (de transporte), en el espa-

\footnotetext{
P. Krugman (1991d).

A. Weber (1909, versión inglesa 1929).
} 
cio, hasta los más complejos de Isard ${ }^{5}$, quien tradujo a términos homogéneos de transporte las formas o funciones de abastecimiento y distribución para determinar la localización óptima con costes mínimos.

Los principales avances consistieron en incorporar a los factores de costes la trascendencia de la demanda, dado sus efectos sobre la localización de la producción. Por otro lado, la estructura de los costes de transporte depende a su vez de ésta. Entre los autores que integraron las variaciones espaciales de la demanda sobresalen Hotteling ${ }^{6}$ y Lösch ${ }^{7}$. Todos estos modelos de localización de la producción se basan en los supuestos de la teoría neoclásica tradicional de información y competencia perfecta, donde la oferta es elástica y tiene rendimientos constantes. El óptimo de la ubicación maximiza los beneficios o ganancias, sujeto a las restricciones de los costes de transporte.

Dentro de este contexto Moses ${ }^{8}$ realizó progresos sustanciales aun sin formalizar los rendimientos crecientes. Su principal objetivo fue integrar la teoría de la localización dentro de una parte de la teoría de la producción en la cual las funciones de producción no son lineales. En ese caso, la cantidad producida puede variar según la demanda y modificar la proporción de los factores empleados. Esto supone importantes implicaciones en los resultados, por cuanto si una función no es lineal no existe un óptimo único, y el de localización puede variar según la demanda debido a la posibilidad de combinaciones diferentes de los factores, las variaciones de sus precios, las de sus calidades y al poder operar a escalas diferentes.

Más recientemente Krugman ${ }^{9}$, como consecuencia de los avances de los modelos de competencia imperfecta en donde se incorporan los rendimientos crecientes, ha formalizado la importancia de éstos y la de los costes de transporte sobre la concentración geográfica de la industria donde se sitúa la demanda final. De este modo, para Krugman, la concentración geográfica de los oferentes y demandantes de inputs industriales se produce por la presencia de rendimientos crecientes, debido a los encadenamientos de demanda hacia adelante y hacia atrás, y la reducción de los costes de transporte. Por otro lado, al desarrollarse un mayor número de proveedores locales especializados, la industria se hace más eficiente, reforzándose la concentración.

\footnotetext{
'W. Isard (1956).

${ }^{\circ}$ H. Hotelling (1929).

A. Lösch (1940).

${ }^{8}$ L. N. Moses (1958).

${ }^{9}$ P. Krugman (1991a, d).
} 
En los trabajos de Krugman, al igual que algunos estudios tradicionales sobre localización industrial, donde destacan los geógrafos ${ }^{10}$, y en cierta forma, también en Lösch ${ }^{11}$, se subrayan otras implicaciones derivadas de la existencia de rendimientos crecientes y/o externalidades económicas. La atención se centra en destacar las economías propias de la localización conjunta de las diferentes industrias en un territorio determinado. Estas economías se denominan de aglomeración y se asocian a los beneficios obtenidos por una empresa individual como resultado de su ubicación en un área de elevada densidad industrial. Se suelen distinguir dos tipos de economías de aglomeración: de localización y de urbanización. Las primeras se consideran internas a la industria a la que pertenecen y se producen en términos de ganancias derivadas de la situación próxima a otras empresas de la misma actividad (o estrechamente ligadas a ella). Las economías de urbanización son típicamente externas a la industria y obtenidas por la totalidad de las empresas que comparten una misma localización, independientemente del sector del cual forman parte.

Las economías de localización son las debidas a las diferentes externalidades consideradas por Marshall ${ }^{12}$. Se pueden distinguir resumidamente tres: en primer lugar, las resultantes de la formación de un mercado local de trabajo cualificado ${ }^{13}$, cuya existencia reduce el riesgo de desempleo a los trabajadores, y al mismo tiempo, ofrece a los empleadores una oferta constante de trabajo, incluso permitiendo mitigar las depresiones económicas al existir mayor diversidad de empresas en la región. En segundo lugar, las derivadas de la oferta de bienes intermedios especializados no comercializables, con la cual existe la posibilidad de la especialización en la producción de inputs para la industria procedentes del propio sector ${ }^{14}$

${ }^{10}$ G. Garofoli (1993), J. P. Gaudemar, J. L. Gaffard y M. Mougeot (1993). Con la consideración de la importancia de los centros o distritos industriales y territorios. También se pueden consultar los trabajos incluidos en el libro de F. Pyke, G. Becattini y W. Sengerberger (1990).

"A. Lösch (1940), pp. 103 a 137. Cuando describe las redes, los cinturones y los sistemas o distritos industriales.

12 A. Marshall (1920).

${ }^{13}$ Paul A. David y Joshua L. Rosenbloom (1990). Se intenta explicar la existencia de las aglomeraciones industriales urbanas mediante un modelo en el que se incorporan los efectos de las externalidades de un mercado de factores marshalliano, tanto de trabajo como de capital.

${ }^{14}$ P. Krugman (1991b, d) ha formalizado, siguiendo a Marshall, los efectos que provoca una industria concentrada en el desarrollo de un mayor número de proveedores de inputs especializados. 
y del sector servicios ${ }^{15}$. Y por último, en tercer lugar, las provocadas por los trasvases tecnológicos, además de la difusión de información («Knowledge or technical spillovers»). Los efectos beneficiosos de la especialización han sido desarrollados también por Arrow (1962) y Romer (1986).

Las economías de urbanización o diversificación consideran que la variedad y diversidad de subsectores próximos geográficamente promueve, debido al aumento de las relaciones interindustriales, la innovación, la difusión de tecnología, de conocimientos y de información ${ }^{16}$, y además, también, la mayor demanda interindustrial por los encadenamientos hacia adelante y hacia atrás, analizados teóricamente por K. MurPhy, A. SHLEIFER, y R. VISHNY (1989) ${ }^{17}$, y, por tanto, el crecimiento industrial. Las externalidades relacionadas con la difusión de ideas y conocimientos ya fueron destacadas por Jacobs (1969) para explicar el crecimiento de las ciudades, por lo que también se les denomina externalidades tipo Jacobs.

De la misma manera se han tenido en cuenta las posibilidades de que los acontecimientos históricos, debido a las ventajas apuntadas, generen un proceso acumulativo, como consecuencia de los rendimientos crecientes, con el resultado de la concentración de una industria o industrias en una determinada región ${ }^{18}$.

A pesar del innegable interés de esta línea de investigación en estos trabajos no se hace referencia a aspectos importantes desde una perspectiva histórica. Previamente al grueso de las investigaciones mencionadas en los párrafos precedentes, sin embargo, Hoover (1948), en La localización de las actividades económicas, ya señaló algunos factores que influyen en la ubicación de determinadas industrias y sus cambios según los diferentes estadios de desarrollo. Según sus conclusiones, en los primeros estadios, la producción se sitúa próxima a las materias primas cuando se trata de

${ }^{15}$ R. Faini (1991). Da más importancia a las implicaciones provocadas por la existencia de un sector terciario que origina efectos inducidos en una región mediante los seivicios ofrecidos a la industria por la importancia de los rendimientos crecientes de este sector en la expansión del sector industrial. Para ello utiliza un modelo con dos regiones y dos sectores: la industria y los servicios.

${ }^{16} \mathrm{Se}$ considera por algunos autores incluso que los efectos indirectos de la difusión de conocimientos alcanzados por esta diversificación son más importantes que la mera concentración geográfica de la producción. D. B. Audretsch y M. P. Feldman (1996), E. L. Glaeser, H. D. Hallal, J. A. Scheinkman y A. Shleifer (1992).

${ }^{17}$ Las consecuencias de las demandas interindustriales son consideradas por estos autores como el principal mecanismo para alcanzar la industrialización cuando se transforman un número suficiente de sectores, al producirse las demandas complementarias entre ellos; de este modo se formalizan las externalidades pecuniarias originadas por los efectos desbordamiento de demanda.

18 P. David (1985) y P. Krugman (1991d). 
los productos intermedios, o cerca de los mercados en los productos acabados. Así ocurrió en el siglo XIX, por ejemplo, en las industrias consumidoras de abundantes materias primas y combustible, como la siderurgia y metalurgia, ubicadas en las cuencas o yacimientos de carbón ${ }^{19}$, o en la industria textil, próxima a los principales mercados, con la creación de centros industriales junto a ellos. En cambio, en las etapas intermedias de desarrollo se produce una dependencia menor de los anteriores factores, la flexibilidad de combinación de materias primas (o distribución de los productos en el mercado) y también la intensidad en el uso de los factores productivos, como considera Moses, aumenta la gama de posibilidades, aunque, según Hoover, se tiende a eliminar las localizaciones intermedias.

El trabajo de Hoover tiene interés también por señalar otros factores más dinámicos con influencia en la localización, como el progreso técnico, el transporte, la energía, y las necesidades de mano de obra y de materias primas ${ }^{20}$. En un país en vías de desarrollo se producirá, primero, una fase vinculada a la tecnología disponible, que en el caso del siglo xIx suponía la utilización de energía procedente del carbón o hidráulica, claro condicionante de la localización industrial por su elevado coste de transporte. En una segunda fase las nuevas innovaciones, con la extensión de la energía eléctrica al uso industrial, posibilitaron el transporte de la energía a larga distancia, favoreciéndose así la difusión de la industria. De este modo, la proximidad a las fuentes de energía pierde importancia en favor de las ubicaciones donde la demanda crece a mayor ritmo. Además de provocar una fragmentación general de la industria y un efecto positivo sobre la industria rural y también familiar, se modificó la estructura misma de los establecimientos respecto de la fábrica del siglo XIX, con necesidad de menos espacio.

A partir de lo anterior, en los siguientes apartados se va a realizar una aproximación a los cambios en la difusión y localización de la industria española en el primer tercio del siglo xx para la comprensión del avance del proceso de industrialización, y por ello, de cómo operaron los distintos efectos que permitieron un desarrollo industrial acumulativo. En general, en el paso de una economía agraria a una industrial es imprescindible la extensión del sector secundario en las propias regiones, con un efecto de atracción hacia las zonas urbanas de la población rural. Pero a su vez también se produce un crecimiento superior de la industria en las regiones

\footnotetext{
19 También en A. Lösch (1940), p. 83.

${ }^{20}$ E. M. Hoover (1948), p. 150.
} 
donde el aumento de la demanda es mayor y donde se originan más ventajas y oportunidades para el desarrollo económico.

\section{LOCALIZACIÓN INDUSTRIAL MANUFACTURERA EN ESPAÑA. LOS DATOS}

Planteados hasta aquí algunos elementos relevantes para el análisis histórico de las teorías de la localización, el objetivo de este apartado es aproximarse al nivel de industria manufacturera per cápita y la estructura de las 50 provincias españolas y sus cambios entre 1913 y 1929. Se ha considerado como unidad espacial la provincia con la finalidad de obtener la mayor desagregación territorial que permiten las fuentes disponibles para valorar los efectos de las externalidades comentadas, y también para conseguir el mayor número de observaciones posibles en el estudio. Se han escogido sólo dos años del período del primer tercio del siglo xx, 1913 y 1929 , debido a la necesidad de construir un indicador de la renta industrial manufacturera regional.

Las fuentes estadísticas españolas no facilitan esta meta aun cuando la trascendencia de ésta para una mejor comprensión de la trayectoria de la economía industrial en esos años sea innegable. Las fuentes posibles para el análisis regional son los impuestos industriales y la población activa empleada en este sector ${ }^{21}$. Ambas tienen inconvenientes para su provecho como indicador de la estructura productiva, pero aun con ellos las ventajas a obtener de su uso son, en mi opinión, muy superiores. Los datos de población activa empleada en los Censos de 1910 y 1930 presentan diferencias en la clasificación que interfieren en la distribución del empleo, entre la agricultura y la industria, así como también en el reducido número de divisiones de éste último. Por lo que en este trabajo se han tenido que utilizar, pues, los impuestos industriales como aproximación a la renta industrial manufacturera. En el caso del período que nos ocupa existe un problema para su empleo, y es que había dos impuestos diferentes. Como es conocido, los impuestos industriales, a partir de 1909, se encontraban divididos en dos contribuciones: la industrial y la de utilidades; en la primera

${ }^{21}$ Las características de estas dos fuentes se pueden consultar en el capítulo 3, y los apéndices del mismo en C. Betrán (1995). Los datos de población activa proceden de los Censos de población. En los impuestos directos, la contribución industrial ha sido utilizada en los estudios industriales para el siglo xx, ver Nadal (1987). A partir de 1909 las sociedades industriales, con determinadas condiciones, pagaban por una nueva contribución, la de utilidades. 
de ellas, de la que hay estadísticas homogéneas desagregadas por subsectores, no están incluidas las provincias sujetas a un régimen fiscal especial, como las del País Vasco y Navarra. En la segunda, en cambio, sí lo están ${ }^{22}$, pero no figuran los datos por subsectores, por lo que se ha tenido que construir una estimación de la contribución que se va a explicar en este apartado.

La contribución de utilidades presenta otro impedimento para su uso como fuente para la economía regional, porque al afectar a empresas de tamaño grande su registro puede no corresponderse con el de la ubicación de la producción de la fábrica o fábricas. Esto sucede con algunas de las sociedades registradas en Madrid, pero también en los centros industriales de Barcelona y Vizcaya, y es más corriente en las empresas químicas, de material eléctrico, construcciones mecánicas, etc. Hay que destacar que por esta causa Madrid tendría una participación importante en la industria en los subsectores de metalurgia, construcción de buques y azúcar. Para obviar esta deficiencia se han distribuido estas sociedades industriales en las distintas unidades de producción atendiendo a la información que sobre ellos se ha podido obtener. Para ello se han utilizado las Estadísticas de Consumo de Carbones, donde se ofrecen datos de producción y consumo de carbón para algunas de estas industrias, las Estadísticas Mineras y Metalúrgicas y las de Producción y Circulación de Azúcares, achicoria y alcohol, y sobre todo la generosa ayuda de un gran número de historiadores económicos ${ }^{23}$.

En el caso de las provincias del País Vasco y Navarra se ha preferido que queden representadas por los datos referentes a sólo una de las contribuciones, la de utilidades, debido a que en ellas se ubica una parte importante y muy significativa, en algunos subsectores, de la industria española, e ignorarla introduciría un mayor sesgo en la explicación de la localización y crecimiento industrial del conjunto nacional. Es evidente, por tanto, la subestimación del País Vasco y Navarra como consecuencia de

${ }^{22}$ Las sociedades anónimas registradas en estas provincias y que tuvieran beneficios producidos por sus operaciones con el resto del país estaban sujetas a la contribución de utilidades. A partir de 1910 , Real Orden de 22 de marzo de 1910 , por lo que afecta a la estimación efectuada en 1913, y en el Real Decreto de 28 de marzo de 1920 , se mantuvo lo anterior, ante la modificación de la contribución de utilidades en ese mismo año, por lo que afecta a la estimación de 1929.

${ }^{23}$ Agradezco la ayuda recibida para realizar este trabajo de Carles Sudrià, Alberto Lozano, José M. Sierra Álvarez, Miguel Gutiérrez, Rafael Uriarte, Nuria Puig y Xavier Tafunell, sin la cual no hubiese podido realizar esta distribución de las sociedades anónimas industriales en los respectivos centros de producción. 
no figurar la contribución industrial, pero, por otro lado, en algunas de ellas las empresas de tamaño grande pertenecientes sobre todo a las industrias de bienes de inversión, de destacado crecimiento en esta etapa, eran muy importantes ${ }^{24}$ (contribuyentes de utilidades) y representaban una parte considerable del total nacional en dichos subsectores, por lo que su omisión plantearía un problema. Se trata de considerar en estas provincias un mínimo de renta industrial manufacturera, donde faltarian las empresas que no eran sociedades anónimas, pero el criterio adoptado permite obtener este dato y controlarlo, frente a la alternativa de excluir una parte relevante de algunas industrias.

La contribución industrial procede de las Estadisticas Administrativas de la Contribución Industrial de 1913 y 1929. Por tanto, en esta parte de la contribución se hace forzoso suponer que el fraude es homogéneo entre subsectores y provincias ${ }^{25}$. Para la Contribución de Utilidades ${ }^{26}$, en las Estadísticas de la Contribución de Utilidades y de la Riqueza Mobiliaria no se ofrece la recaudación clasificada en los diferentes tipos de industria como en la primera, lo cual ha obligado a elaborar una estimación. El método empleado para calcular la contribución de utilidades hipotética ha sido su reconstrucción a partir de las sociedades sujetas a ésta y una estimación de los beneficios industriales para cada uno de los años mencionados ${ }^{27}$. Las sociedades consideradas son las anónimas, las cuales eran las únicas contribuyentes en 1913; pero, en cambio, el resto de asociaciones sí podían hacerlo desde 1922. Sin embargo, como existía un régimen de opción ${ }^{28} \mathrm{y}$, por tanto, la posibilidad de continuar pagando por la industrial, cabe suponer que muchas sociedades colectivas, las más abundantes entre los nuevos contribuyentes, siguieran con la misma contribución.

Las sociedades sujetas al nuevo impuesto y su capital desembolsado se han obtenido para 1913 de las Estadísticas de la Contribución de Utilidades de dicho año, aunque ha sido necesario completarlas a partir de otras fuentes y agruparlas en las diferentes ramas industriales. Y para 1929, al no existir una relación como la anterior, se ha conseguido de los Anuarios Financieros y de Sociedades Anónimas (AFSA). Como ya se ha mencionado, en esta información se han corregido las sociedades cuyo lugar de registro

${ }^{21}$ Como sería el caso de Vizcaya, y menos en el resto. Ver J. Catalán (1990).

${ }^{25}$ Sobre la validez del supuesto se puede consultar J. Nadal y X. Tafunell (1992), pp. $255-260$.

${ }^{26}$ La tarifa tercera, como en el caso de la contribución industrial, era la que pagaba la industria.

27 La información sobre su elaboración está a disposición de los investigadores.

${ }^{2 *}$ Ley de 29 de abril de 1920, disposición cuarta del artículo tercero. 
no coincide con el de producción o bien se encuentra repartido en varias fábricas, a través de indicadores indirectos de la importancia relativa de cada una de éstas.

La estimación de los beneficios parte de la obtención, para cada empresa industrial de la que se tiene esta información, de una tasa próxima a la de rentabilidad (la relación entre los beneficios y el capital desembolsado) procedente de los AFSA y los Anuarios Financieros de Bilbao. Con ellas se ha calculado para cada año una tasa de rentabilidad media ponderada por el capital desembolsado de estas empresas. Si se hace el supuesto de que la relación anterior es representativa de todo el sector industrial, se pueden estimar los beneficios de cada subsector en relación a su capital desembolsado. De este modo se ha considerado una tasa de rentabilidad única para el conjunto de la industria para conseguir una mayor proximidad a la estructura industrial, dado que la obtención de mayores beneficios por parte de algunos subsectores por el grado de monopolio en el mercado podría influir en su peso, así como evita las diferencias en la información sobre las empresas de cada subsector.

La contribución de utilidades se ha calculado mediante la aplicación de los tipos de gravámenes existentes en cada año a los beneficios subsectoriales estimados. En 1913 este gravamen era fijo, del 6 por 100, y en 1929 ya era progresivo y dependía de la relación entre los beneficios y el capital desembolsado, por lo que según la escala vigente se ha aplicado la misma tarifa para todos los subsectores. Al ser progresivo el gravamen y crecer el sector secundario, en 1929, respecto a 1913, las tasas de crecimiento del impuesto son altas. De este modo, en el segundo año la contribución de utilidades tiene más peso.

Los subsectores en que se ha podido establecer la suma de las dos contribuciones son 20: aceite, bebidas, alimentación, azúcar, harinero, textil, cuero, calzado y sombreros, artes gráficas, papelero, cemento, cerámica y vidrio, madera y mueble, corcho, automóvil, buques, metalurgia, transformados metálicos, material eléctrico, químico, caucho y varias ${ }^{29}$. Se trata, por tanto, de una parte de la industria, la manufacturera, con la exclusión de los subsectores energéticos.

Por otro lado, y en relación con la población, las diferencias destacadas en su tamaño entre regiones hacen necesario medir la importancia de la industria manufacturera en términos por habitante. En el cuadro 1 figuran

${ }^{24}$ La composición de los impuestos industriales de los 20 subsectores procedentes de las contribuciones industriales y de utilidades están a disposición de los investigadores que lo soliciten. 


\section{CUADRO 1}

Impuestos industriales manufactureros provinciales, en 1913 y 1929

\begin{tabular}{|c|c|c|c|c|c|c|c|}
\hline Num. & Provincias & $\begin{array}{c}\text { IND/CAP } \\
1913\end{array}$ & $C$ & $\begin{array}{c}I N D / C A P \\
1929\end{array}$ & $C$ & $\begin{array}{c}T A S A \\
\text { (porcentaje) }\end{array}$ & $C$ \\
\hline 1 & Álava & 0,52 & 19 & 2,04 & 17 & 8,85 & 17 \\
\hline 2 & Albacete ........ & 0,23 & 41 & 0,96 & 29 & 9,33 & 13 \\
\hline 3 & 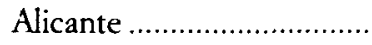 & 0,53 & 18 & 2,12 & 16 & 8,99 & 16 \\
\hline 4 & 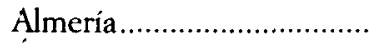 & 0,18 & 44 & 0,41 & 47 & 5,13 & 43 \\
\hline 5 & Ávila. & 0,28 & 34 & 0,92 & 31 & 7,65 & 24 \\
\hline 6 & Badajoz .......... & 0,27 & 37 & 0,72 & 41 & 6,25 & 37 \\
\hline 7 & Baleares... & 0,40 & 24 & 1,60 & 23 & 9,01 & 15 \\
\hline 8 & 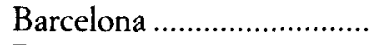 & 4,08 & 1 & 13,95 & 1 & 7,99 & 22 \\
\hline 9 & 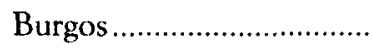 & 0,31 & 31 & 1,70 & 20 & 11,15 & 6 \\
\hline 10 & 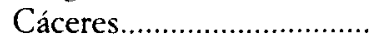 & 0,23 & 42 & 0,45 & 46 & 4,34 & 47 \\
\hline 11 & 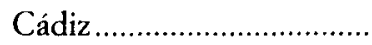 & 0,96 & 8 & 2,00 & 18 & 4,71 & 45 \\
\hline 12 & 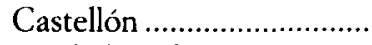 & 0,27 & 36 & 1,35 & 27 & 10,52 & 9 \\
\hline 13 & Ciudad Real ............................. & 0,34 & 28 & 0,56 & 44 & 3,11 & 49 \\
\hline 14 & Córdoba .......... & 0,35 & 27 & 1,30 & 28 & 8,54 & 19 \\
\hline 15 & 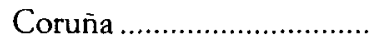 & 0,28 & 35 & 0,84 & 34 & 7,19 & 26 \\
\hline 16 & 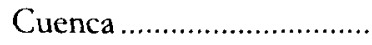 & 0,19 & 43 & 0,71 & 42 & 8,64 & 18 \\
\hline 17 & 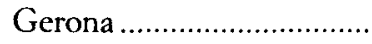 & 1,27 & 5 & 2,84 & 10 & 5,18 & 42 \\
\hline 18 & Granada. & 0,84 & 10 & 2,24 & 14 & 6,36 & 36 \\
\hline 19 & Guadalajara.. & 0,27 & 38 & 1,49 & 25 & 11,27 & 5 \\
\hline 20 & Guipúzcoa.... & 1,30 & 4 & 7,92 & 3 & 11,97 & 3 \\
\hline 21 & Huelva........ & 0,67 & 15 & 0,73 & 40 & 0,57 & 50 \\
\hline 22 & Huesca ............................... & 0,13 & 47 & 2,40 & 11 & 19,73 & 1 \\
\hline 23 & 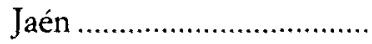 & 0,30 & 32 & 0,91 & 32 & 7,18 & 27 \\
\hline 24 & Las Palmas..... & 0,16 & 46 & 0,76 & 36 & 10,24 & 10 \\
\hline 25 & León ................. & 0,29 & 33 & 0,74 & 39 & 6,10 & 39 \\
\hline 26 & Lérida ............... & 0,60 & 17 & 1,61 & 22 & 6,40 & 35 \\
\hline 27 & 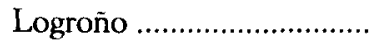 & 0,42 & 22 & 3,54 & 7 & 14,18 & 2 \\
\hline 28 & 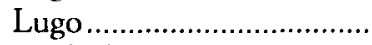 & 0,09 & 48 & 0,17 & 50 & 4,51 & 46 \\
\hline 29 & Madrid. & 1,2 & 6 & 5,30 & 4 & 9,71 & 11 \\
\hline 30 & Málaga.... & 0,69 & 14 & 1,79 & 19 & 6,17 & 38 \\
\hline 31 & Murcia............ & 0,27 & 39 & 0,92 & 30 & 8,08 & 21 \\
\hline 32 & 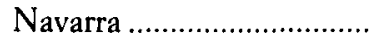 & 0,39 & 25 & 2,26 & 13 & 11,63 & 4 \\
\hline 33 & Orense & 0,05 & 50 & 0,22 & 49 & 9,55 & 12 \\
\hline 34 & 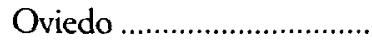 & 1,1 & 7 & 3,28 & 9 & 7,06 & 28 \\
\hline 35 & 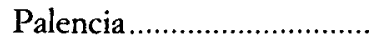 & 0,33 & 29 & 0,76 & 37 & 5,33 & 41 \\
\hline 36 & 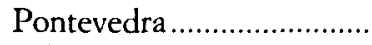 & 0,24 & 40 & 0,56 & 43 & 5,41 & 40 \\
\hline 37 & 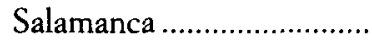 & 0,41 & 23 & 0,76 & 38 & 3,88 & 48 \\
\hline 38 & 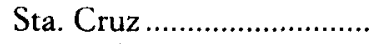 & 0,07 & 49 & 0,23 & 48 & 8,10 & 20 \\
\hline 39 & 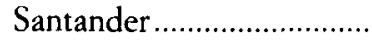 & 0,94 & 9 & 4,91 & 6 & 10,90 & 7 \\
\hline
\end{tabular}




\begin{tabular}{|c|c|c|c|c|c|c|c|}
\hline Núm. & Provincias & $\begin{array}{c}I N D / C A P \\
1913\end{array}$ & $C$ & $\begin{array}{c}I N D / C A P \\
1929\end{array}$ & $C$ & $\begin{array}{c}\text { TASA } \\
\text { (porcentaje) }\end{array}$ & $C$ \\
\hline 40 & Segovia... & 0,47 & 21 & 1,50 & 24 & 7,55 & 25 \\
\hline 41 & Sevilla........ & 0,73 & 13 & 2,12 & 15 & 6,90 & 30 \\
\hline 42 & 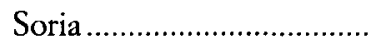 & 0,32 & 30 & 0,86 & 33 & 6,41 & 33 \\
\hline 43 & Tarragona ............................... & 0,79 & 12 & 2,31 & 12 & 6,92 & 29 \\
\hline 44 & Teruel........ & 0,49 & 20 & 1,39 & 26 & 6,74 & 32 \\
\hline 45 & Toledo.......... & 0,37 & 26 & 0,80 & 35 & 5,01 & 44 \\
\hline 46 & Valencia.............................. & 0,82 & 11 & 3,33 & 8 & 9,13 & 14 \\
\hline 47 & 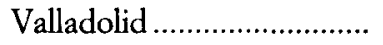 & 0,62 & 16 & 1,67 & 21 & 6,40 & 34 \\
\hline 48 & Vizcaya ................................ & 2,18 & 2 & 10,91 & 2 & 10,60 & 8 \\
\hline 49 & 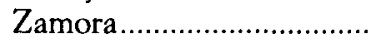 & 0,17 & 45 & 0,48 & 45 & 6,82 & 31 \\
\hline 50 & Zaragoza............................... & 1,48 & 3 & 5,00 & 5 & 7,89 & 23 \\
\hline & TOTAL. & 0,77 & & 3,04 & & 8,97 & \\
\hline
\end{tabular}

FUENTE: Elaboración propia.

NOTA: IND/CAP, Impuestos industria manufacturera por habitante en millones de pesetas constantes de 1913. C, clasificación, ordenación decreciente; TASA, tasa de crecimiento media anual acumulativa, en porcentajes; $\mathrm{C}$, clasificación, ordenación decreciente. Se ha deflactado el año 1929 en pesetas constantes de 1913 a través del deflactor implícito del valor añadido industrial elaborado a partir de A. Carreras (1982).

los impuestos industriales de las manufacturas por habitante de las distintas provincias en 1913 y 1929, así como su tasa de crecimiento anual acumulativa entre ambos años. En 1913 Barcelona tenía la preeminencia $(4,08$ pts. de 1913/hab.), seguida de Vizcaya (2,18 ptas. de 1913/hab.), Zaragoza $(1,48)$, Guipúzcoa $(1,30)$ y Gerona $(1,27)$. Madrid en 1913 se encontraba en una sexta posición en cuanto a nivel de industrialización. A pesar de ello, recuperó posiciones en 1929, pasando al cuarto lugar. Tal vez en este año Guipúzcoa figura en un puesto inferior al que le corresponde respecto a Zaragoza como consecuencia de que en esta provincia, junto a las del resto del País Vasco y Navarra, persiste el problema derivado de la inexistencia de la contribución industrial.

En 1929 destacaron por el siguiente orden: Barcelona (13,95 ptas. de 1913/hab.), Vizcaya (10,91 ptas. de 1913/hab.), Guipúzcoa (7,92), Madrid $(5,30)$ y Zaragoza $(5,00)$. En relación al nivel de industria manufacturera per cápita las provincias que crecieron por encima de la media $(8,97$ por $100)$ fueron, por orden descendente: Huesca $(19,73)$, Logroño $(14,18)$, Guipúzcoa (11,97), Navarra (11,63 por 100), Guadalajara $(11,27)$, Burgos $(11,15)$, Santander $(10,90)$, Vizcaya $(10,60)$, Castellón $(10,52)$, Las Palmas 
$(10,24)$, Madrid $(9,71)$, Orense $(9,55)$, Albacete $(9,33)$, Valencia $(9,13)$, Baleares $(9,01)$ y Alicante $(8,99)$.

En definitiva, se puede comprobar cómo cinco de las seis provincias con mayor industria manufacturera por habitante son las mismas en 1913 y en 1929, aunque con distinta importancia relativa. Las provincias con un nivel superior a la media española en $1913(0,77)$ eran 12. Además de las mencionadas figuraban Oviedo $(1,10)$, Cádiz $(0,96)$, Santander $(0,94)$, Granada $(0,84)$, Valencia $(0,82)$ y Tarragona $(0,79)$. En cambio, en 1929, por encima de la media $(3,04)$ estuvieron sólo nueve provincias: a Zaragoza $(5,00)$ le siguieron Santander $(4,91)$, Logroño $(3,54)$, Valencia $(3,33)$ y Oviedo $(3,28)$.

\section{DIFUSIÓN DE LAS TRANSFORMACIONES INDUSTRIALES MANUFACTURERAS: MEDICIÓN DE LA ESPECIALIZACIÓN}

En el apartado anterior se ha constatado la relevancia industrial manufacturera de cada provincia en 1913 y 1929. Ahora cabe también preguntarse si se produjo entre estos dos años un aumento en la especialización provincial; con otras palabras, si las provincias se fueron especializando según sus ventajas competitivas, e incluso por las razones de que los rendimientos crecientes pueden hacer favorable la especialización independientemente de las condiciones iniciales de éstas ${ }^{30}$. O si, en cambio, por las transformaciones en España hacia la diversificación en la estructura del sector secundario, por el avance del desarrollo de la industrialización, ha ocurrido, en general, todo lo contrario: una reducción o mantenimiento de la especialización industrial manufacturera por la difusión de los fenómenos constatados en el conjunto nacional con considerables implicaciones favorables en el crecimiento futuro.

Cuando la industrialización tiene lugar se produce un aumento de la participación de los bienes intermedios industriales empleados por otros sectores, y esto sólo sucede cuando la industria se hace más compleja y se forma un tejido industrial previo. Posteriormente, cuando una economía está más desarrollada tiene lugar un aumento de la concentración, en ciu-

${ }^{30}$ P. Krugman (1991d, trad. castell. 1992), p. 12. Comenta cómo se le ha concedido a la idea de Ricardo las ventajas comparativas de un énfasis excesivo cuando los rendimientos crecientes pueden explicar el comercio y el intercambio de productos incluso en países que parten de condiciones económicas muy similares. 
dades y distritos, de características marcadamente diferentes ${ }^{31}$, a menos que ocurran algunos cambios relevantes favorecedores de la dispersión, como especialmente los que aumentan la movilidad de los factores productivos, en las regulaciones económicas e incluso por la congestión y la saturación del centro industrial ${ }^{32}$.

Para contrastar todo ello se va a emplear, en primer lugar, un índice de la divergencia entre las estructuras industriales manufactureras regionales, en este caso provinciales, respecto a la media nacional. En otros términos, este procedimiento permite observar la incidencia regional de las transformaciones industriales a nivel nacional mediante una aproximación cuantitativa. El índice del grado de especialización provincial es el siguiente,

$$
\mathrm{G}_{\mathrm{p}}=\frac{1}{2} \sum_{i=1}^{n}\left|\mathrm{~s}_{i}^{p}-\mathrm{s}_{i}^{n}\right|
$$

siendo $\mathrm{s}_{i}^{p}$ y $\mathrm{s}_{i}^{n}$ la participación de la manufactura $i, i=1, \ldots, 20$, en el total del sector en la provincia $p, p=1, \ldots, 50$ y en la nación $n$, respectivamente. De forma que cuando la provincia y el total nacional tengan idénticas estructuras el índice tomará el valor 0 , y cuando sean completamente diferentes (al tener en cuenta los pesos de los subsectores de forma íntegra) alcanzará el valor 1.

Para su estimación se ha comparado la estructura industrial manufacturera de cada provincia respecto al promedio nacional en 1913 y 1929 , resultados resumidos en el cuadro $2^{33}$. Se puede observar cómo en general

${ }^{31}$ P. Krugman (1991, trad. castell. 1992), pp. 59 y 60. Explica que esto ocurrió en Estados Unidos a finales del siglo XIX, cuando ya era una economía industrial. La economía se hizo más compleja y diversa, de forma que el porcentaje de productos industriales empleados en la producción aumentó, produciéndose las condiciones favorables para la formación de complejos y distritos industriales, porque en ellos existen los proveedores de productos intermedios especializados.

${ }^{32}$ Ruth C. Young (1986). También en S. Kim (1995) se argumenta que la concentración en Estados Unidos alcanzó el grado más alto en el período de entreguerras y posteriormente cayó sustancial y continuamente.

${ }^{3}$ Nótese que aunque pudiera parecer por la importancia de los impuestos industriales por habitante en algunas provincias, como: Barcelona, Madrid y Vizcaya, que representan una participación mayoritaria en el total nacional que definiera la estructura industrial nacional, esto no ocurre así, pues en términos de la distribución del impuesto total, en 1913, estas tres provincias representan el $30,38,6,90$ y 4,97 por 100 , respectivamente, de la industria nacional, y en 1929 , el $35,05,10,24$ y 7,39 por 100 . Además sus estructuras industriales 


\section{CUADRO 2}

Índice del grado de especialización, en 1913 y 1929 (indice y clasificación)

\begin{tabular}{|c|c|c|c|c|c|c|}
\hline \multirow[b]{2}{*}{ Núm. } & \multirow[b]{2}{*}{ Provincias } & \multicolumn{2}{|c|}{ Año 1913} & \multicolumn{2}{|c|}{ Año 1929} & \multirow{2}{*}{$-\begin{array}{c}\text { Tasa } \\
\text { (porcentaje) }\end{array}$} \\
\hline & & Indice & Clasificación & Indice & Clasificación & \\
\hline 1 & Álava...... & 0,74 & 2 & 0,77 & 1 & 0,31 \\
\hline 2 & Albacete.............................. & 0,51 & 27 & 0,56 & 20 & 0,50 \\
\hline 3 & 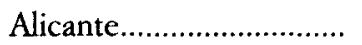 & 0,16 & 50 & 0,31 & 47 & 4,31 \\
\hline 4 & Almería ...................... & 0,52 & 25 & 0,63 & 9 & 1,23 \\
\hline 5 & Ávila............ & 0,59 & 11 & 0,64 & 6 & 0,54 \\
\hline 6 & Badajoz....... & 0,54 & 22 & 0,68 & 3 & 1,46 \\
\hline 7 & Baleares ..... & 0,31 & 47 & 0,27 & 50 & $-0,82$ \\
\hline 8 & Barcelona.......... & 0,39 & 39 & 0,29 & 49 & $-1,75$ \\
\hline 9 & 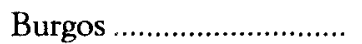 & 0,51 & 26 & 0,48 & 31 & $-0,37$ \\
\hline 10 & 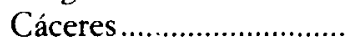 & 0,56 & 16 & 0,52 & 26 & $-0,45$ \\
\hline 11 & 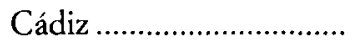 & 0,66 & 3 & 0,68 & 2 & 0,18 \\
\hline 12 & 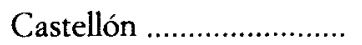 & 0,37 & 42 & 0,40 & 41 & 0,47 \\
\hline 13 & Ciudad Real.. & 0,64 & 6 & 0,63 & 8 & $-0,05$ \\
\hline 14 & 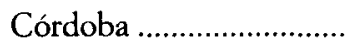 & 0,37 & 40 & 0,47 & 33 & 1,46 \\
\hline 15 & 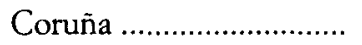 & 0,49 & 32 & 0,46 & 36 & $-0,42$ \\
\hline 16 & 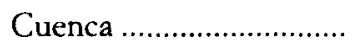 & 0,55 & 19 & 0,57 & 17 & 0,22 \\
\hline 17 & 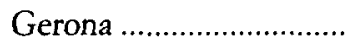 & 0,42 & 37 & 0,48 & 32 & 0,82 \\
\hline 18 & Granada..... & 0,65 & 4 & 0,61 & 13 & $-0,43$ \\
\hline 19 & Guadalajara .... & 0,58 & 13 & 0,67 & 4 & 0,88 \\
\hline 20 & 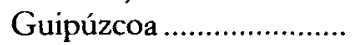 & 0,55 & 20 & 0,53 & 25 & $-0,25$ \\
\hline 21 & 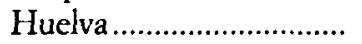 & 0,50 & 31 & 0,55 & 22 & 0,59 \\
\hline 22 & 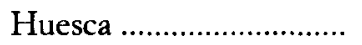 & 0,48 & 33 & 0,54 & 24 & 0,72 \\
\hline 23 & 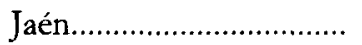 & 0,53 & 24 & 0,52 & 29 & $-0,13$ \\
\hline 24 & 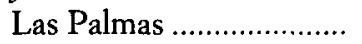 & 0,54 & 23 & 0,61 & 14 & 0,77 \\
\hline 25 & 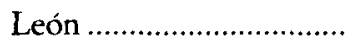 & 0,50 & 30 & 0,52 & 27 & 0,25 \\
\hline 26 & 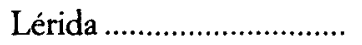 & 0,45 & 35 & 0,47 & 34 & 0,23 \\
\hline 27 & 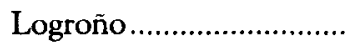 & 0,37 & 41 & 0,64 & 7 & 3,46 \\
\hline 28 & 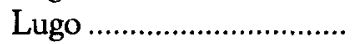 & 0,56 & 18 & 0,54 & 23 & $-0,15$ \\
\hline 29 & Madrid........... & 0,34 & 44 & 0,36 & 46 & 0,33 \\
\hline 30 & Málaga ................................. & 0,30 & 49 & 0,37 & 44 & 1,36 \\
\hline 31 & Murcia ................................ & 0,34 & 45 & 0,39 & 42 & 0,89 \\
\hline 32 & 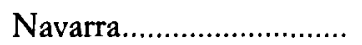 & 0,78 & 1 & 0,62 & 10 & $-1,38$ \\
\hline 33 & 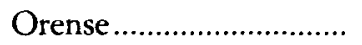 & 0,60 & 9 & 0,61 & 12 & 0,16 \\
\hline
\end{tabular}

son muy diferentes, como se puede observar en los cuadros del texto. E incluso, por ejemplo, en el caso de Barcelona, el textil supone en el total de su industria el 57,72 por 100 en 1913 y el 46,46 por 100 en 1929 , cuando este subsector en el total de la media nacional tiene una participación diferente del 23,09 por 100 en 1913 y del 19,59 por 100 en 1929. 


\begin{tabular}{|c|c|c|c|c|c|c|}
\hline \multirow[b]{2}{*}{ Núm. } & \multirow[b]{2}{*}{ Provincias } & \multicolumn{2}{|c|}{ Año 1913} & \multicolumn{2}{|c|}{ Año 1929} & \multirow{2}{*}{$\begin{array}{c}\text { Tasa } \\
\text { (porcentaje }\end{array}$} \\
\hline & & Indice & Clasificación & Indice & Clasificación & \\
\hline 34 & Oviedo.......... & 0,57 & 15 & 0,55 & 21 & $-0,17$ \\
\hline 35 & Palencia.. & 0,56 & 17 & 0,60 & 15 & 0,45 \\
\hline 36 & Pontevedra... & 0,60 & 8 & 0,52 & 28 & $-0,84$ \\
\hline 37 & Salamanca .......................... & 0,57 & 14 & 0,45 & 37 & $-1,48$ \\
\hline 38 & Sta. Cruz .. & 0,51 & 28 & 0,50 & 30 & $-0,10$ \\
\hline 39 & Santander ............... & 0,36 & 43 & 0,43 & 38 & 1,23 \\
\hline 40 & 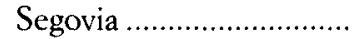 & 0,64 & 5 & 0,57 & 16 & $-0,70$ \\
\hline 41 & 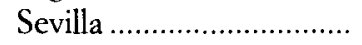 & 0,39 & 38 & 0,46 & 35 & 1,14 \\
\hline 42 & Soria ......................... & 0,59 & 12 & 0,62 & 11 & 0,35 \\
\hline 43 & 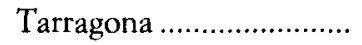 & 0,30 & 48 & 0,36 & 45 & 1,18 \\
\hline 44 & Teruel ................................ & 0,63 & 7 & 0,65 & 5 & 0,22 \\
\hline 45 & Toledo .............................. & 0,54 & 21 & 0,56 & 18 & 0,23 \\
\hline 46 & Valencia ............................. & 0,31 & 46 & 0,30 & 48 & $-0,27$ \\
\hline 47 & 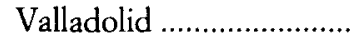 & 0,44 & 36 & 0,43 & 39 & $-0,25$ \\
\hline 48 & Vizcaya & 0,48 & 34 & 0,39 & 43 & $-1,34$ \\
\hline 49 & 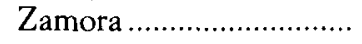 & 0,60 & 10 & 0,56 & 19 & $-0,42$ \\
\hline 50 & 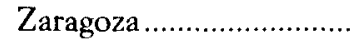 & 0,51 & 29 & 0,42 & 40 & $-1,13$ \\
\hline & MEDIA. & 0,499 & & 0,516 & & \\
\hline & MEDIA PONDERADA... & 0,439 & & 0,395 & & \\
\hline
\end{tabular}

FUENTE: Elaboración propia, como se explica en el texto.

NoTA: ÍNDICE, Índice del grado de especialización provincial, comprendido entre 0 y 1. CLASIFICACIÓN, ordenación descendente. TASA, tasa de crecimiento media anual acumulativa en porcentajes.

se ha producido una disminución de 21 índices de especialización regional, $y$ otras provincias que los han elevado los mantienen reducidos entre estos dos años. La media y la media ponderada por el tamaño de la industria de cada provincia de los índices de especialización nos muestra esta tendencia: en 1913 la medias eran de 0,499 y 0,439 , respectivamente, y en 1929 la primera se estabiliza en 0,516 y la segunda disminuye a 0,395 . Por otra parte, el coeficiente de variación también disminuyó entre estos años, pasó del 0,24 al 0,22. Esto significa que no aumentó la especialización regional entre 1913 y 1929. Por tanto, en su mayoría las estructuras productivas de las regiones no cambiaron hacia una mayor especialización, siguieron la evolución del conjunto nacional.

Las provincias más especializadas en 1913 eran Navarra $(0,78)$, Álava 
$(0,74)^{34}$, Cádiz $(0,66)$, Granada $(0,65)$ y Segovia $(0,64)$. En 1929 , en cambio, figuraban en primer lugar Álava $(0,77)$, seguida de Cádiz $(0,68)$, Badajoz $(0,68)$, Guadalajara $(0,67)$ y Teruel $(0,65)$. Por contra, entre las provincias menos especializadas, con una estructura más diversificada, fueron las principales: en 1913, Alicante $(0,16)$, Málaga $(0,30)$, Tarragona $(0,36)$, Baleares $(0,31)$ y Valencia $(0,31)$. En 1929 en las primeras posiciones estaban Baleares $(0,27)$, Barcelona $(0,29)$, Valencia $(0,30)$, Alicante $(0,31)$ y Madrid $(0,36)$, aunque Alicante con un índice de especialización un poco más elevado que en 1913. A partir de ello se puede concluir que en esta etapa del proceso de industrialización las transformaciones en el sector secundario se difundieron de forma que la mayoría de las provincias disminuyeron o mantuvieron su grado de especialización.

\section{FUENTES DEL CRECIMIENTO INDUSTRIAL MANUFACTURERO REGIONAL, 1913-1929}

Este apartado tiene el propósito de analizar el efecto de la especialización y la diversificación del sector secundario en el crecimiento industrial en las diferentes provincias entre 1913 y 1929. La diversificación puede tener dos implicaciones positivas en el crecimiento en un país todavía no industrializado: el aumento de la demanda interindustrial mediante los encadenamientos hacia delante y hacia atrás ${ }^{35} \mathrm{y}$ favorecer, por la formación de un tejido industrial más complejo, la difusión de conocimientos, información, nuevas aplicaciones tecnológicas y la adopción del cambio técnico. En definitiva, en la primera se aumenta el tamaño del mercado de la región con la obtención de externalidades pecuniarias, al poder reducir los costes y los precios; en la segunda se deriva el efecto de las externalidades tecnológicas o economías externas entre las distintas industrias, en este caso denominadas tipo Jacobs (knowledge spillovers).

Por otro lado, también se va a estudiar la importancia de las denominadas economías de especialización debido a las diferentes externalidades consideradas por Marshall, dado que, como se ha señalado, éstas pudieron tener importancia en la concentración de una industria en una determinada región, y de ahí en su crecimiento. De este modo, el estudio regional permite

${ }^{3+}$ Hay que tener en cuenta que ello puede ocurrir en estas dos provincias, porque sólo se utiliza la contribución de utilidades.

is Se pueden consultar los efectos en la industria espanola durante el primer tercio del siglo xx en C. Betrán (1997). 
tener más observaciones y distinguir los diversos factores que incidieron en la expansión de esta etapa ${ }^{36}$. Para ello se ha realizado un análisis econométrico, en el cual se intenta mostrar la relevancia que en el crecimiento industrial manufacturero per cápita de las regiones, en este caso a nivel provincial, entre 1913 y 1929, tuvieron las condiciones de: el nivel de industria manufacturera per cápita, la especialización y la diversificación manufacturera de las provincias en el año de partida, 1913. Del siguiente modo,

$$
\mathrm{I}_{t / t-1}=c+\alpha_{1} \mathrm{I}_{t-1}+\alpha_{2} \mathrm{ESP}_{t-1}+\alpha_{3} \mathrm{DI}_{t-1}+\varepsilon_{t}
$$

siendo $t-1=1913$ y $t=1929$.

Donde la variable dependiente $\mathrm{I}_{t / t-1}$ es el crecimiento de la industria manufacturera per cápita (impuestos directos per cápita) de cada provincia entre 1913 y 1929, y las variables explicativas: $\mathrm{I}_{t-1}$, la industria manufacturera por habitante inicial en 1913; $\mathrm{ESP}_{t-1}$, el índice de especialización en 1913; $\mathrm{DI}_{t-1}$. el índice de diversificación en 1913. La muestra tiene 50 observaciones.

La especialización se ha medido mediante el índice construido en el apartado anterior, a partir de la diferencia en la participación de cada rama manufacturera en el sector de la provincia en relación a la del total nacional. Mide pues, cuán de especializada está una provincia en relación a lo que se esperaría si siguiese el comportamiento nacional. Su valor está comprendido entre el valor 0 y 1 , desde la menor a la mayor especialización. La diversificación se ha obtenido mediante dos índices sobre la variedad de ramas manufactureras existentes en cada provincia: el primero, un índice de diversificación de Hirschman-Herfindal ${ }^{37}$ (el sumatorio del cuadrado de la participación de cada rama manufacturera en el sector de una provincia). Está contenido entre el valor 0 y 1, desde la máxima a la mínima diversificación. El segundo se define como la proporción de la industria manufacturera, que representan los cinco subsectores más importantes ${ }^{38}$.

${ }^{36}$ Del tipo de los efectuados por E. L. Glaeser, et al. (1992), V. Henderson (1994) y J. A. Herce, et al. (1996). La diferencia en este caso es que se ha considerado como unidad de observación la región o provincia, en lugar del subsector, debido a que el objetivo es estudiar el grado de difusión de los cambios en la estructura industrial manufacturera en la economía española del periodo y evaluar algunos de los factores explicativos del crecimiento industrial mediante un análisis regional. Este análisis permite obtener mayores observaciones sobre el avance industrial y la importancia de los efectos de desbordamiento de demanda medidos a través de la diversificación de la estructura productiva que tiene lugar en un proceso de industrialización.

${ }^{37}$ Utilizado por Henderson (1994) para medir la diversificación.

${ }^{38}$ Utilizado por Glaeser, et al. (1992), para medir la diversificación industrial. 
Como el anterior, está acotado entre el valor 0 y 1 , desde la mayor a la menor diversificación.

Mediante este análisis se pretende también discriminar entre la importancia que tuvieron las economías de localización o especialización, externalidades marshallianas, y las de urbanización o diversificación, también denominadas externalidades tipo Jacobs. Un signo positivo (negativo) del coeficiente del índice de especialización mostraría un efecto positivo (negativo) de la especialización en el crecimiento industrial de las distintas provincias, y lo contrario, en el caso de la diversificación, un signo negativo (positivo) significa un efecto positivo (negativo), porque un menor (mayor) valor del índice expresa mayor (menor) diversificación. Por otro lado, el coeficiente del valor inicial (1913) de la industria por habitante indicaría, si es negativo (positivo), que las industrias que partían de un menor valor inicial han convergido (divergido) a las de mayor valor de esta variable una vez se ha controlado por el resto de variables independientes consideradas.

\subsection{Descripción de los datos}

En los cuadros 3.1, 3.2 y 4.1, 4.2 se presentan los ejemplos de la distribución de los cinco principales subsectores en las cinco provincias de

\section{CUADRO 3.1}

Principales subsectores de las cinco provincias con mayor industria manufacturera por babitante en 1913

\begin{tabular}{ll}
\hline Barcelona & Textil $(57,72)$, Transf. Metálicos $(8,38)$, Químico (4,86), Varias \\
& $(4,04)$ y Artes Gráficas $(3,48)$. \\
Vizcaya & Metalúrgica $(24,82)$, Buques $(21,83)$, Textil $(16,18)$, Transf. Metá- \\
& licos $(10,88)$ y Bebidas $(6,22)$. \\
Zaragoza & Azúcar $(53,79)$, Químico $(12,00)$, Harinero $(8,37)$, Transf. Metá- \\
& licos $(4,79)$ y Textil $(3,33)$. \\
Guipúzcoa & Papel $(25,78)$, Transf. Metálicos $(16,71)$, Químico (16,35), Varias \\
Gerona & $(11,89)$ y Alimentación $(7,49)$. \\
& Textil $(50,28)$, Harinero $(8,66)$, Corcho $(8,24)$, Madera y mueble \\
& $(6,53)$ y Papel $(6,41)$.
\end{tabular}

FUENTE: Elaboración propia.

NOTA: Se han indicado solamente los cinco mayores subsectores; entre paréntesis figura su participación en porcentajes sobre el total en cada provincia. 
mayor y menor industria manufacturera por habitante en 1913 y 1929. Se observa, tanto en 1913 como en 1929, que en las provincias con mayor nivel de industria manufacturera por habitante tienen más importancia las industrias de bienes intermedios y de equipo que en las de menor nivel, donde los subsectores de bienes de consumo final: harina, azúcar, madera y mueble, aceite, cuero y calzado, cerámica y alimentación representan una parte destacada del total de la industria manufacturera.

\section{CUADRO 3.2}

Principales subsectores de las cinco provincias con mayor industria manufacturera por babitante en 1929

$\begin{array}{ll}\text { Barcelona } & \text { Textil }(46,46), \text { Transformados Metálicos }(10,12), \text { Química }(10,11), \\ & \text { Cemento, cerámica y vidrio }(4,86), \text { Alimentación }(3,43) . \\ \text { Vizcaya } & \text { Metalúrgica }(29,07), \text { Transformados Metálicos }(18,49), \text { Química } \\ & (11,95), \text { Cerámica y vidrio }(6,79), \text { Buques }(5,41) . \\ \text { Guipúzcoa } & \text { Transformados Metálicos }(30,40), \text { Papel }(24,66), \text { Química }(16,54), \\ & \text { Artes Gráficas }(9,13) \text {, Alimentación }(5,55) . \\ \text { Madrid } & \text { Transformados Metálicos }(15,79), \text { Química }(14,39) \text {, Artes Gráficas } \\ & (11,58), \text { Material eléctrico }(10,19) \text {, Cemento, cerámica y vidrio } \\ \text { Zaragoza } & (9,89) . \\ & \text { Azúcar }(45,46), \text { Química }(14,33), \text { Transf. Metálicos }(8,21), \text { Bebi- } \\ & \text { das }(6,93), \text { Cerámica y vidrio (5,61). }\end{array}$

FUENTE: Elaboración propia.

NotA: Se han indicado solamente los cinco mayores subsectores; entre paréntesis figura su participación en porcentajes sobre el total en cada provincia.

Por otro lado, tiene interés comentar la especialización regional. Para ello, se ha empleado el coeficiente de especialización regional, definido como el cociente entre la participación de cada manufactura en el total del sector en una provincia respecto a la participación de dicha manufactura en el total del sector nacional. Se puede expresar el coeficiente de especialización del subsector $i$ en la provincia $p$ como,

$$
\mathrm{E}_{i}^{p}=\frac{s_{i}^{p}}{s_{i}^{n}}
$$




\section{CUADRO 4.1}

Principales subsectores de las cinco provincias con menor industria manufacturera por babitante en 1913

\begin{tabular}{|c|c|}
\hline Orense & $\begin{array}{l}\text { Harinero }(32,75) \text {, Alimentos }(19,28) \text {, Madera y mueble }(18,88) \text {, } \\
\text { Artes Gráficas }(8,61) \text { y Cuero y calzados }(5,05) \text {. }\end{array}$ \\
\hline Sta. Cruz & $\begin{array}{l}\text { Harinero }(33,93) \text {, Buques }(20,98) \text {, Madera y mueble }(7,35) \text {, Ali- } \\
\text { mentación }(7,20) \text { y Bebidas }(7,11) .\end{array}$ \\
\hline Lugo & $\begin{array}{l}\text { Harinero }(34,87) \text {, Alimentos }(19,38) \text {, Cuero y calzado }(9,76) \text {, } \\
\text { Madera y Mueble }(8,58) \text { y Artes Gráficas }(5,79) \text {. }\end{array}$ \\
\hline Huesca & $\begin{array}{l}\text { Harinero (33,03), Alimentos }(15,05) \text {, Aceite }(12,22) \text {, Químico } \\
\quad(7,42) \text { y Cemento, cerámica y vidrio }(7,11) \text {. }\end{array}$ \\
\hline Las Palmas & $\begin{array}{l}\text { Varias }(32,89) \text {, Harinero }(14,53) \text {, Alimentación }(9,93) \text {, Metalurgia } \\
\quad(9,40) \text { y Buques }(7,45) \text {. }\end{array}$ \\
\hline
\end{tabular}

FUENTE: Elaboración propia.

Nota: Se han indicado solamente los cinco mayores subsectores; entre paréntesis figura su participación en porcentajes sobre el total en cada provincia.

siendo,

$$
\begin{aligned}
& s_{i}^{p}=\frac{x_{i}^{p}}{x^{p}} \\
& s_{i}^{n}=\frac{x_{i}^{n}}{x^{n}}
\end{aligned}
$$

$\mathrm{x}_{i}^{p}, \mathrm{x}_{i}^{n} \mathrm{y} \mathrm{x}^{p}, \mathrm{x}^{n}$ son los impuestos de la manufactura $i$ en la provincia $p$ y la nación, y el total de los impuestos de la provincia y la nación; siendo, en este caso, $p=1, \ldots, 50$, e $i=1, \ldots, 20$. De esta manera, un coeficiente igual a uno significa que la participación de una manufactura en la provincia es igual a la del conjunto nacional, y si es mayor (menor) a la unidad en la provincia la importancia de ésta es mayor (menor) que en el total nacional.

Los resultados obtenidos para las cinco provincias con mayor y menor industria manufacturera por habitante se pueden ver en los cuadros 5.1, 5.2 y 6.1, 6.2. Se han ordenado, según la importancia de los coeficientes de especialización y se muestran sólo los mayores que 1 . Entre los ejemplos 
CUADRO 4.2

Principales subsectores de las cinco provincias con menor industria manufacturera por babitante en 1929

\begin{tabular}{|c|c|}
\hline Lugo & $\begin{array}{l}\text { Harina }(24,19) \text {, Alimentación }(20,93) \text {, Madera y mueble }(20,30) \text {, } \\
\text { Transformados Metálicos }(9,72) \text {, Cemento, cerámica y vidrio } \\
(6,56) \text {. }\end{array}$ \\
\hline Orense & $\begin{array}{l}\text { Madera y mueble }(38,54) \text {, Cemento, cerámica y vidrio }(16,34) \text {, } \\
\text { Harina }(12,71) \text {, Alimentación }(10,50) \text {, Transformados Metálicos } \\
(6,17) \text {. }\end{array}$ \\
\hline Sta. Cruz & $\begin{array}{c}\text { Varias }(15,87) \text {, Harina }(13,93) \text {, Cemento, cerámica y vidrio } \\
(10,78) \text {, Alimentación }(10,67) \text {, Artes Gráficas }(10,53) .\end{array}$ \\
\hline Almería & $\begin{array}{l}\text { Azúcar }(34,23) \text {, Varias }(21,59) \text {, Harina }(15,06) \text {, Aceite }(7,98) \text {, } \\
\text { Cemento, cerámica y vidrio }(5,06) \text {. }\end{array}$ \\
\hline Cáceres & $\begin{array}{l}\text { Harina }(29,05) \text {, Aceite }(27,89) \text {, Químico }(12,76) \text {, Cemento, cerá- } \\
\text { mica y vidrio }(5,89) \text {, Bebidas }(5,19) \text {. }\end{array}$ \\
\hline
\end{tabular}

FUENTE: Elaboración propia.

Nota: Se han indicado solamente los cinco mayores subsectores; entre paréntesis figura su participación en porcentajes sobre el total en cada provincia.

más característicos cabe resaltar a Barcelona por su especialización (su participación es mucho más alta que el promedio) hacia industrias que, en un principio, pueden tener fuertes implicaciones en el crecimiento económico, como las de bienes intermedios y de equipo. Así, entre éstas en 1913 figuran: automóvil, material eléctrico, textil, caucho, varias y transformados metálicos, y en 1929 se encuentran el textil, automóvil, cuero y calzado, material eléctrico, varias y transformados metálicos. Por otro lado, también destacaría Madrid con una gran variedad, donde figuran también los subsectores mencionados con: artes gráficas, caucho, transformados metálicos, madera y mueble, cemento, cerámica y vidrio, bebidas, varias y material eléctrico, en 1913. Y en 1929, material eléctrico, automóvil, caucho, varias, transformados metálicos, cemento, cerámica y vidrio, cuero y calzado, madera y mueble y químico.

Sin embargo, algunas provincias se especializan en industrias con menores repercusiones en el resto, basadas sobre todo en la elaboración de productos agrícolas, como Orense (madera y mueble, alimentación, harina, cuero, pieles y calzado y artes gráficas) o Lugo (cuero y calzado, alimentación, harina, madera y muebles), así como en 1929 en Almería (varias, azúcar, harina, aceite y corcho) o Cáceres (aceite, harina, varias, madera 


\section{CUADRO 5.1}

Especialización manufacturera de las cinco provincias con mayor industria manufacturera por habitante en 1913

\begin{tabular}{|c|c|}
\hline rcel & $\begin{array}{l}\text { Automóvil }(3,04) \text {, Material Eléctrico }(2,58) \text {, Textil }(2,50) \text {, Caucho } \\
(2,30) \text {, Varias }(1,17) \text {, Transformados metálicos }(1,16) \text {. }\end{array}$ \\
\hline zcava & $\begin{array}{l}\text { Buques }(16,42) \text {, Metalúrgica }(5,49) \text {, Papel }(2,69) \text {, Transformados } \\
\text { Metálicos }(1,50) \text {, Cemento, cerámica y vidrio }(1,03) \text {. }\end{array}$ \\
\hline 2 & \\
\hline ul & $\begin{array}{l}\text { Varias }(3,43) \text {, Transformados Metálicos }(2,30) \text {, Quí- } \\
\text { Alimentación }(1,73) \text {, Madera y mueble }(1,29) \text {. }\end{array}$ \\
\hline erc & $\begin{array}{l}\text { Corcho }(21,27) \text {, Papel }(2,99) \text {, Textil }(2,18) \text {, Madera y mueble } \\
(1,54) \text {. }\end{array}$ \\
\hline
\end{tabular}

FUENTE: Elaboración propia.

NotA: En el cuadro aparecen sólo los subsectores manufactureros con coeficientes de especialización mayores que 1 , que figuran entre paréntesis.

y mueble y caucho). Valencia, en cambio, al igual que Alicante, se especializa en subsectores de bienes de consumo, pero con una amplia variedad: la madera y el mueble, papel, químico, cuero y calzado, bebidas, cemento,

\section{CUADRO 5.2}

Especialización manufacturera de las cinco provincias con mayor industria manufacturera por babitante en 1929

\begin{tabular}{|c|c|}
\hline lona & $\begin{array}{l}\text { Textil }(2,37) \text {, Automóvil }(1,41) \text {, Cuero y calzado }(1,31) \text {, Material } \\
\text { Eléctrico }(1,15) \text {, Varias }(1,08) \text {, Transformados Metálicos }(1,04) \text {. }\end{array}$ \\
\hline 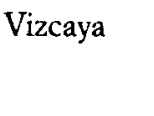 & $\begin{array}{l}\text { Metalúrgica }(4,63) \text {, Buques }(2,77) \text {, Transf. Metálicos }(1,90) \text {, Papel } \\
(1,50) \text {, Artes Gráficas }(1,48) \text {, Cemento, cerámica y vidrio }(1,09) \text {, } \\
\text { Bebidas }(1,06) \text {. }\end{array}$ \\
\hline úzcoa & $\begin{array}{c}\text { Papel }(10,07) \text {, Transformados metálicos }(3,12) \text {, Artes Gráficas } \\
(2,50) \text {, Químico }(1,27) \text {, Alimentación }(1,20) \text {, Varias }(1,01) .\end{array}$ \\
\hline$\therefore d$ & $\begin{array}{l}\text { Material Eléctrico }(3,63) \text {, Automóvil }(3,25) \text {, Caucho }(2,98) \text {, Varias } \\
\text { (2,51), Transformados Metálicos }(1,62) \text {, Cemento, cerámica y } \\
\text { vidrio }(1,58) \text {, Cuero y calzado }(1,47) \text {, Madera y mueble }(1,38) \text {, } \\
\text { Químico }(1,11) \text {. }\end{array}$ \\
\hline & ebidas $(1,26)$, Química $(1$, \\
\hline
\end{tabular}

FUENTE: Elaboración propia.

Nota: En el cuadro aparecen sólo los subsectores manufactureros con coeficientes de especialización mayores que 1 , que figuran entre paréntesis. 


\section{CUADRO 6.1}

Especialización manufacturera de las cinco provincias con menor industria manufacturera por babitante en 1913

\begin{tabular}{|c|c|}
\hline rense & $\begin{array}{l}\text { Madera y mueble }(4,61) \text {, Alimentación }(4,44) \text {, Harina }(3,17) \text {, Cue } \\
\text { ro y calzado }(2,34) \text {, Artes Gráficas }(2,13) \text {. }\end{array}$ \\
\hline a. Cruz & $\begin{array}{l}\text { Buques }(15,78) \text {, Harinero }(3,28) \text {, Madera y mueble }(1,79) \text {, Ali } \\
\text { mentación }(1,66) \text {, Cemento, cerámica y vidrio }(1,20) \text {, Artes Grá } \\
\text { ficas }(1,16) .\end{array}$ \\
\hline 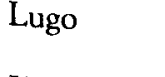 & $\begin{array}{l}\text { Cuero y Calzado (4,52), Alimentación }(4,46) \text {, Harina }(3,38) \\
\text { Madera y Mueble }(2,09) \text {, Varias }(1,63) \text {, Artes Gráficas }(1,43)\end{array}$ \\
\hline ue & $\begin{array}{l}\text { Alimentación }(3,47) \text {, Harinero }(3,20) \text {, Aceite }(3,14) \text {, Artes Gráfic } \\
(1,70) \text {, Cemento, cerámica y vidrio }(1,54) \text {, Madera y muel } \\
(1,25) \text {, Químico }(1,02) \text {. }\end{array}$ \\
\hline Las Palmas & $\begin{array}{l}\text { Varias }(9,49) \text {, Buques }(5,60) \text {, Automóviles }(3,66) \text {, Alimentac } \\
(2,29) \text {, Metalúrgico }(2,08) \text {, Harinero }(1,41) \text {, Madera y mue } \\
(1,34) \text {. }\end{array}$ \\
\hline
\end{tabular}

FUENTE: Elaboración propia.

Nota: En el cuadro aparecen sólo los subsectores manufactureros con coeficientes de especialización mayores que 1 , que figuran entre paréntesis.

\section{CUADRO 6.2}

Especialización manufacturera de las cinco provincias con menor industria manufacturera por babitante en 1929

Lugo

Orense

Sta. Cruz

Almería

Cáceres
Madera y mueble (5,41), Harinero (4,58), Alimentación (4,54), Caucho $(1,79)$, Artes Gráficas $(1,38)$, Cuero y calzado $(1,31)$, Cemento, cerámica y vidrio $(1,05)$, Transformados Metálicos (1).

Madera y Mueble $(10,28)$, Cemento, cerámica y vidrio $(2,62)$, Cuero y calzado $(2,46)$, Harina $(2,41)$, Alimentación $(2,27)$, Artes Gráficas $(1,06)$.

Varias $(8,17)$, Artes Gráficas $(2,88)$, Harina $(2,64)$, Alimentación $(2,31)$, Cuero y calzado $(1,93)$, Cerámica y vidrio $(1,72)$.

Varias (11,11), Azúcar $(5,87)$, Harina $(2,85)$, Aceite $(2,79)$, Corcho $(1,77)$.

Aceite $(9,75)$, Harina $(5,51)$, Varias (2,59), Madera y Mueble $(1,14)$, Caucho $(1,05)$.

FUENTE: Elaboración propia.

Nota: En el cuadro aparecen sólo los subsectores manufactureros con coeficientes de especialización mayores que 1 , que figuran entre paréntesis. 
cerámica y vidrio, harina, varias, aceite, transformados metálicos y artes gráficas. Vizcaya se centra en las industrias de bienes de inversión: buques, metalúrgica, papel, transformados metálicos, cemento, cerámica y vidrio.

En los cuadros 7.1, 7.2 y 8.1 y 8.2 figuran las cinco provincias más y menos especializadas ${ }^{39}$. Las provincias, en 1913 , eran Navarra $(0,78)$ (azúcar y papel), Álava $(0,73)$ (azúcar) ${ }^{40}$ y Granada $(0,65)$ (azúcar), Cádiz $(0,66)$ (bebidas) y Segovia $(0,64)$ (química, cemento, cerámica y vidrio y harina). En 1929, Navarra $(0,62)$ redujo considerablemente su especialización ${ }^{41}$ y fue una de las provincias de mayor crecimiento. Granada y Segovia también la redujeron; sin embargo, Álava y Cádiz la aumentaron $(0,77$ y 0,68 , respectivamente). Álava, en 1929 , se especializa en el azúcar, los transformados metálicos y la harina ${ }^{42}$. Después de Álava tomaron el relevo en los primeros puestos Cádiz, Badajoz, Guadalajara y Teruel, en las que en la primera, además de las bebidas, destacaba en buques, harinas y varias; en las siguientes, Badajoz y Teruel, en industrias derivadas de la agricultura, y por otro lado, Guadalajara con el automóvil, harina, químico $\mathrm{y}$ aceite.

\section{CUADRO 7.1}

Especialización manufacturera de las cinco provincias más especializadas en 1913

Navarra Azúcar (10,36), Papel $(2,92)$.

Álava Azúcar $(10,33)$.

Cádiz Bebidas (9,35).

Granada Azúcar $(9,19)$.

Segovia

Químico $(7,15)$, Cerámica y vidrio $(2,87)$, Harina $(2,07)$.

FUENTE: Elaboración propia.

Nota: En el cuadro aparecen sólo los subsectores manufactureros con coeficientes de especialización mayores que 1 , que figuran entre paréntesis.

${ }^{39}$ Se sigue el indice de especialización calculado en el apartado 3.

${ }^{40}$ En estas dos provincias hay que tener en cuenta que sólo se ha considerado la contribución de utilidades, por tanto, a las sociedades anónimas. La omisión de la contribución industrial puede afectar al índice de especialización obtenido. Entre los subsectores que tendrían participación si se contase con la contribución que recaía sobre el resto de las sociedades figurarían la harina, la alimentación y la madera y mueble.

${ }^{41}$ Aunque en este resultado puede influir el menor nivel de partida en el primer año de las sociedades no anónimas que en el segundo año se convirtiesen en anónimas y figurasen en su subsector correspondiente tributando por la contribución de utilidades.

${ }^{+2}$ Según los datos disponibles, pero, como se ha mencionado, se han dejado de considerar las sociedades no anónimas. 


\section{CUADRO 7.2}

\section{Especialización manufacturera de las cinco provincias más especializadas en 1929}

\begin{tabular}{ll}
\hline Álava & Azúcar $(6,13)$, Transf. Metálicos $(4,99)$, Harina $(2,61)$, \\
Cádiz & Bebidas $(9,90)$, Buques $(9,30)$, Harina $(1,53)$, Varias $(1,06)$, \\
Badajoz & Alimentación $(5,80)$, Bebidas $(4,31)$, Harina $(3,43)$, Aceite $(3,29)$, \\
& Madera y Mueble $(3,07)$. \\
Guadalajara & Automóvil $(19,10)$, Harina $(3,56)$, Químico $(2,40)$, Aceite $(2)$. \\
Teruel & Azúcar $(9,63)$, Aceite $(5,11)$, Madera y Mueble $(1,58)$, Harina \\
& $(1,09)$.
\end{tabular}

FUENTE: Elaboración propia.

Nota: En el cuadro aparecen sólo los subsectores manufactureros con coeficientes de especialización mayores que 1, que figuran entre paréntesis.

\section{CUADRO 8.1}

\section{Especialización manufacturera de las cinco provincias menos especializadas en 1913}

\begin{tabular}{|c|c|}
\hline Alicante & $\begin{array}{l}\text { Papel }(3,67) \text {, Cuero y Calzado }(1,87) \text {, Aceite }(1,32) \text {, Químico } \\
(1,32) \text {, Cerámica y vidrio }(1,28) \text {, Varias }(1,11) \text {, Harina }(1,09) \text {, } \\
\text { Textil }(1,08) \text {, Madera y mueble }(1,05) \text {. }\end{array}$ \\
\hline Málaga & $\begin{array}{l}\text { Azúcar }(2,51) \text {, Bebidas }(2,13) \text {, Aceite }(2,11) \text {, Metalúrgico }(1,81) \text {, } \\
\text { Harinero }(1,13) \text {. }\end{array}$ \\
\hline Tarragona & Aceite $(3,63)$, Bebidas $(2,63)$, Químico $(1,97)$. \\
\hline Baleares & $\begin{array}{l}\text { Cuero y Calzado }(4,41) \text {, Alimentación }(2,40) \text {, Transf. Metálicos } \\
(2,20) \text {, Madera y mueble }(1,63) \text {, Químico }(1,53) \text {, Harinero } \\
(1,08) \text {, Textil }(1,05) \text {. }\end{array}$ \\
\hline Valencia & $\begin{array}{l}\text { Madera y mueble }(2,12) \text {, Papel }(1,88) \text {, Químico }(1,77) \text {, Cuero } \\
\text { y calzado }(1,73) \text {, Bebidas }(1,61) \text {, Cemento, cerámica y vidrio } \\
\text { (1,57), Harinero }(1,50) \text {, Varias }(1,39) \text {, Aceite }(1,37) \text {, Transfor- } \\
\text { mados Metálicos }(1,50) \text {, Artes Gráficas }(1,12) \text {. }\end{array}$ \\
\hline
\end{tabular}

FUENTE: Elaboración propia.

Nota: En el cuadro aparecen sólo los subsectores manufactureros con coeficientes de especialización mayores que 1 , que figuran entre paréntesis.

En estos cuadros también se resalta que las provincias con un índice de especialización más elevado lo están en pocos subsectores (con elevados coeficientes); sin embargo, las menos lo están en más, con una gran variedad 


\section{CUADRO 8.2 \\ Especialización manufacturera de las cinco provincias menos especializadas en 1929}

\begin{tabular}{|c|c|}
\hline Baleares & $\begin{array}{l}\text { Cuero y calzado }(6,62) \text {, Caucho }(5,44) \text {, Madera y mueble }(2,15) \text {, } \\
\text { Alimentación }(1,52) \text {, Bebidas }(1,39) \text {, Químico }(1,33) \text {, Cerámica } \\
\text { y vidrio }(1,22) \text {, Harina }(1,18) \text {. }\end{array}$ \\
\hline Barcelona & $\begin{array}{l}\text { Textil }(2,37) \text {, Automóvil }(1,41) \text {, Cuero y calzado }(1,31) \text {, Material } \\
\text { Eléctrico }(1,15) \text {, Varias }(1,08) \text {, Transformados Metálicos }(1,04) \text {. }\end{array}$ \\
\hline Valencia & $\begin{array}{l}\text { Buques }(3,56) \text {, Metalúrgico }(3,42) \text {, Madera y Mueble }(1,83) \text {, } \\
\text { Cemento, cerámica y vidrio }(1,54) \text {, Alimentación }(1,36) \text {, Aceite } \\
(1,31) \text {, Caucho }(1,15) \text {, Bebidas }(1,07) \text {. }\end{array}$ \\
\hline Alicante & $\begin{array}{c}\text { Papel }(3,25) \text {, Cuero y calzado }(2,20) \text {, Aceite }(2,10) \text {, Bebidas }(1,81) \text {, } \\
\text { Varias }(1,70) \text {, Textil }(1,57) \text {, Harina }(1,21) \text {, Químico }(1,15) \text {. }\end{array}$ \\
\hline Madrid & $\begin{array}{l}\text { Material Eléctrico }(3,63) \text {, Automóvil }(3,25) \text {, Caucho }(2,98) \text {, Varias } \\
(2,51) \text {, Transformados Metálicos }(1,62) \text {, Cemento, cerámica y } \\
\text { vidrio }(1,58) \text {, Cuero y calzado }(1,47) \text {, Madera y mueble }(1,38) \text {, } \\
\text { Químico }(1,11) \text {. }\end{array}$ \\
\hline
\end{tabular}

FUENTE: Elaboración propia.

Nota: En el cuadro aparecen sólo los subsectores manufactureros con coeficientes de especialización mayores que 1 , que figuran entre paréntesis.

de subsectores (con coeficientes más bajos), tanto de bienes de consumo como intermedios industriales y de equipo. Ello nos muestra que los indicadores de especialización y diversificación en estos años, sobre todo en 1913, están correlacionados.

\subsection{Resultados del crecimiento industrial manufacturero por habitante provincial en 1913-1929}

Como se ha enunciado en el apartado anterior, se ha realizado una estimación econométrica de la importancia en el crecimiento industrial manufacturero por habitante de las provincias, entre 1913 y 1929, de la especialización y diversificación ${ }^{43}$. Para ello, el método de estimación ha sido el de mínimos cuadrados ordinarios, corregidos por heteroscedasti-

${ }^{43}$ A sugerencia de uno de los evaluadores, se ha efectuado también un análisis utilizando como unidad de observación el subsector. Las resultados obtenidos muestran el mismo signo de los coeficientes de las variables, aunque no son significativas. Una de las explicaciones de la no significatividad puede ser el que se ha tenido que eliminar, más de un 
cidad. A continuación se explica cómo se han obtenido e interpretado éstos.

En primer lugar, en la estimación (1) la variable explicativa considerada es el nivel de industria manufacturera por habitante inicial. Esta variable tiene un coeficiente con signo negativo, aunque no es significativa ${ }^{44}$. Pero los datos confeccionados tienen el inconveniente de presentar errores de medida en el caso de las provincias vascas y Navarra como consecuencia de que para ellas sólo se ha podido considerar la contribución de utilidades que recae sobre las sociedades anónimas. De este modo cuando se incluye una dummy, llamada DPV, para las provincias anteriores, ésta [estimación (2)] es significativa y con signo positivo y el signo del coeficiente de la industria por habitante en 1913 se mantiene, aunque todavía no llega a ser significativo.

El ajuste mejora con la introducción de las variables referentes a la especialización y diversificación para el año inicial. Se trata de analizar el posible efecto de la mayor especialización y/o diversificación provincial en el crecimiento de la industria manufacturera por habitante entre 1913 y 1929. Las variables de especialización y diversificación mencionadas, para esta última con las dos medidas construidas, no son significativas conjuntamente, al existir correlación entre ellas. De forma que cuando la especialización fue alta (baja), la diversificación, construida de modo que cuando es menor el valor del índice mayor (menor) es la diversificación, la mayoría de las veces también lo hizo así. Por ello, se han tenido que incluir separadamente en las ecuaciones, puesto que una variable anula parte del efecto de la otra por la multicolinealidad.

En el cuadro 9 la estimación (3), donde se emplean como variables explicativas la especialización en 1913, con la dummy DPV anterior, convierte a la industria manufacturera por habitante inicial en significativa

30 por 100 de la muestra, por no existir observaciones de algunos subsectores en algunas provincias en el año inicial. Hay que considerar además la posible disminución de la calidad de los mismos al bajar al detalle subsectorial, pues se trata de una aproximación a la renta industrial manufacturera a través de los impuestos industriales, los cuales, a su vez, proceden de una estimación (sólo en el caso de la contribución de utilidades).

${ }^{44}$ La estimación de esta ecuación que presenté al II Encuentro de Historia Económica, en la UIMP, Valencia, 1997, con los datos de impuestos sin corregir por las sociedades que tenian sus centros de producción en diferentes provincias a las de su registro, el signo era positivo, y cambiaba cuando se incluía una dummy para las provincias más ricas. La explicación es que las provincias de Barcelona, Vizcaya y Madrid tenían en 1913 y 1929 un nivel de industria manufacturera per cápita mayor que el resto y se encontraban entre las de mayor crecimiento por esa causa. El que ahora la dummy anterior no sea significativa muestra la mejora obtenida en la confección de los datos con la corrección. 


\section{CUADRO 9}

Crecimiento de la industria manufacturera por habitante, 1913-1929

\begin{tabular}{|c|c|c|c|c|c|c|c|c|}
\hline \multicolumn{9}{|l|}{$\operatorname{In}\left(I_{1929} / I_{1913}\right)$} \\
\hline Variables & (1) & (2) & (3) & (4) & (5) & (6) & (7) & (8) \\
\hline Constante..... & $\begin{array}{c}1,17 \\
(15,31)\end{array}$ & $\begin{array}{c}1,09 \\
(14,50)\end{array}$ & $\begin{array}{c}1,76 \\
(9,69)\end{array}$ & $\begin{array}{c}1,69 \\
(10,33)\end{array}$ & $\begin{array}{c}1,29 \\
(12,12)\end{array}$ & $\begin{array}{c}1,28 \\
(12,03)\end{array}$ & $\begin{array}{c}2,50 \\
(7,16)\end{array}$ & $\begin{array}{c}2,33 \\
(8,05)\end{array}$ \\
\hline $\operatorname{lnI}_{1913} \ldots \ldots \ldots$ & $\begin{array}{l}-0,03 \\
(-0,40)\end{array}$ & $\begin{array}{l}-0,075 \\
(-1,03)\end{array}$ & $\begin{array}{l}-0,16 \\
(-1,98)\end{array}$ & $\begin{array}{r}-0,095 \\
(-1,87)\end{array}$ & $\begin{array}{l}-0,07 \\
(-0,99)\end{array}$ & $\begin{array}{c}-0,02 \\
(-0,37)\end{array}$ & $\begin{array}{l}-0,12 \\
(-1,57)\end{array}$ & $\begin{array}{l}-0,06 \\
(-1,23)\end{array}$ \\
\hline $\operatorname{ESP}_{1913} \ldots \ldots \ldots$ & & & $\begin{array}{c}-1,53 \\
(-3,90)\end{array}$ & $\begin{array}{l}-1,35 \\
(-4,02)\end{array}$ & & & & \\
\hline $\mathrm{DIV}_{1913} \ldots \ldots \ldots$ & & & & & $\begin{array}{c}-0,91 \\
(-3,29)\end{array}$ & $\begin{array}{c}-0,81 \\
(-3,11)\end{array}$ & & \\
\hline $\operatorname{DIN}_{1913 \ldots \ldots . .}$ & & & & & & & $\begin{array}{l}-1,84 \\
(-3,93)\end{array}$ & $\begin{array}{l}-1,59 \\
(-4,19)\end{array}$ \\
\hline DPV .................. & & $\begin{array}{r}0,534 \\
(4,43)\end{array}$ & $\begin{array}{c}0,83 \\
(6,35)\end{array}$ & $\begin{array}{c}0,79 \\
(6,41)\end{array}$ & $\begin{array}{c}0,71 \\
(6,28)\end{array}$ & $\begin{array}{c}0,68 \\
(6,15)\end{array}$ & $\begin{array}{c}0,76 \\
(6,48)\end{array}$ & $\begin{array}{c}0,72 \\
(6,45)\end{array}$ \\
\hline $\mathrm{D} 22 \ldots \ldots \ldots$ & & & & $\begin{array}{c}1,65 \\
(22,08)\end{array}$ & & $\begin{array}{c}1,70 \\
(21,78)\end{array}$ & & $\begin{array}{c}1,62 \\
(20,34) \\
\end{array}$ \\
\hline $\mathrm{R}^{2}$ ajust......... & 0,003 & 0,10 & 0,24 & 0,49 & 0,17 & 0,44 & 0,25 & 0,50 \\
\hline$N$ (obs.)......... & 50 & 50 & 50 & 50 & 50 & 50 & 50 & 50 \\
\hline
\end{tabular}

Nota: Método de estimación MCO corregidos por heteroscedasticidad (Método de White). El estadístico $t$ figura entre paréntesis debajo de cada coeficiente.

VARLABLE: variable endógena $(\mathrm{en} \ln ), \ln \left(\mathrm{I}_{1929} / \mathrm{I}_{1913}\right)$, el crecimiento de la industria manufacturera por habitante; las variables explicativas $\ln I_{1913}$, industria manufacturera por habitante en 1913 (en ln); $\mathrm{ESP}_{1913}$, indice de especialización en 1913; $\mathrm{DIV}_{1913}$, primer índice de diversificación; DIN $_{1913}$, segundo índice de diversificación, que considera la participación de los cinco primeros subsectores. Las dummies, DPV, dummy de las provincias del País Vasco y Navarra (núms. 1, 20, 48 y 32), D22, dummy de Huesca.

manteniendo el signo negativo; por tanto, el signo esperado. De este modo, cuando se controla por la especialización, las provincias con un nivel de industria manufacturera más bajo crecieron más que las más industrializadas por término medio. Por lo que si se espera que haya cierta relación entre el nivel de industria manufacturera per cápita alcanzado por una provincia y su grado de especialización o diversificación por el propio avance del proceso de industrialización, debido a la mayor diversificación de las estructuras manufactureras cuando aumenta la variedad de subsectores, al considerar las dos variables conjuntamente se analiza el efecto parcial de cada una de ellas. Los resultados nos muestran que la especialización es muy significativa y tiene el signo negativo. Éste y el elevado coeficiente de la 
especialización indica que ésta no debió tener un efecto favorable en el crecimiento ${ }^{45}$.

En la estimación (4) se incluye una dummy para Huesca, una provincia de escasa importancia industrial, pero de crecimiento considerable gracias al desarrollo del subsector eléctrico en los Pirineos y su efecto en la localización de algunas industrias. Se puede observar en el cuadro 9 la elevada significatividad y aumento del $\mathrm{R}^{2}$ conseguido con su presencia.

Y por último, en la estimación (5) se considera la diversificación medida mediante el primer indicador. Esta variable es significativa y de signo negativo. La interpretación, dado que un índice de diversificación más bajo (alto) indica mayor (menor) diversificación, es, por tanto, que las provincias más diversificadas en 1913 tuvieron tasas mayores de crecimiento industrial. En esta ocasión el nivel de industria manufacturera por habitante inicial no es significativo, aunque presenta el signo negativo. La inclusión de la dummy para Huesca en la estimación (6) mejora el ajuste y tiene un signo positivo y es significativa. Los mismos resultados se obtienen si utilizamos la segunda variable para medir la diversificación como se comprueba en las estimaciones (7) y (8). Aunque, en cambio, aumenta la significatividad de la industria manufacturera por habitante de 1913, el coeficiente de la variable diversificación es mucho más alto y el ajuste también es mejor.

En resumen, los resultados obtenidos apuntan que el efecto positivo en la explicación del crecimiento fue el producido por la diversificación en la estructura industrial, por su impacto en el tamaño del mercado para el sector secundario y por las fuertes externalidades, como la divulgación de información, conocimiento, difusión tecnológica, etc. Este efecto debió ser mucho más importante, porque se trata de un período de avance industrial, de cambio estructural, dentro de una etapa del proceso de industrialización que finalizó en la década de los sesenta y, por tanto, en el que el sector industrial todavía tenía un peso reducido en la economía.

\section{CONCLUSIONES}

En un proceso de industrialización se originan destacados cambios en la composición del sector secundario hacia una mayor importancia de las

${ }^{45}$ Se podría contemplar también la estimación considerando si las provincias que se especializaron más a lo largo del período de 1913 y 1929 crecieron más; por tanto, incluir la variable ESPt, siendo $t$ el año 1929, una vez de nuevo se controla por el nivel inicial de industria manufacturera per cápita. Si se realiza la estimación los resultados obtenidos son parecidos. La especialización no tuvo un efecto positivo en el crecimiento de este período. 
industrias de bienes intermedios y de equipo. En el período de 1913 y 1929 tuvo lugar en España uno de estos avances. Este avance significó una superior capacidad de la economía española para producir bienes más complejos debido a los cambios en la oferta: tecnología, acumulación de capital físico y de capital humano, así como de la demanda, hacia bienes de elasticidad-renta positiva. Con la finalidad de destacar el alcance y la relevancia de algunas de estas transformaciones se ha realizado en este trabajo un análisis regional. Para lo cual ha sido necesario hacer una estimación de la renta industrial manufacturera y su composición a partir de la reconstrucción de los impuestos directos para las 50 provincias españolas y 20 manufacturas.

En primer lugar, no se produjo un aumento de la especialización de la industria manufacturera entre 1913 y 1929, de forma que la diversificación del conjunto nacional debió afectar a la mayoría de las provincias. Esta evolución tuvo repercusiones importantes en el crecimiento posterior, dado que muestra un avance del proceso de industrialización y de la potencialidad de aprovecharse de las externalidades propias de la difusión de información, tecnología, cercanía a los proveedores de inputs industriales, tanto de bienes intermedios como de bienes de equipo, incluso el desarrollo de otro tipo de infraestructuras procedentes del sector servicios: subsector financiero, redes de transporte, entre otras. Así como una prueba de la importancia que pudieron tener los efectos desbordamiento de demanda en el crecimiento del sector industrial.

En segundo lugar, las estimaciones realizadas indican que las provincias más diversificadas crecieron más entre 1913 y 1929, por lo que la diversificación fue favorable para el crecimiento. En cambio, no lo fue la especialización, que en buena parte se trataba de industrias manufactureras relacionadas con el sector primario, por consiguiente, con menor impacto para inducir el desarrollo del sector manufacturero, que todavía tenía un tamaño reducido en estas regiones. De forma que en esta etapa fueron más importantes las relaciones interindustriales (externalidades tipo Jacobs) que las intraindustriales (externalidades marshalianas) en el crecimiento industrial. En definitiva, por tanto, del mismo modo la formación de un tejido industrial más complejo e interrelacionado, como ocurrió entre 1913 y 1929, debió tener una gran trascendencia en el aumento del tamaño del sector secundario en la economía y entonces en la continuación del proceso de industrialización español.

La distinción de las provincias más especializadas, tanto en 1913 como en 1929, da ejemplo de que éstas lo estaban mucho en muy pocos sub- 
sectores cuando lo contrario ocurría en las provincias menos especializadas en los mismos años. Además, sucede que las mencionadas provincias menos especializadas, entre la variedad de subsectores, se encontraban los bienes intermedios y de equipo. Estos subsectores debieron tener una notable relevancia como difusores de las externalidades positivas explicadas en el trabajo.

\section{BIBLIOGRAFÍA}

ARRow, K. J. (1962): «The Economics implications of Learning by Doing», en Review of Economic Studies, núm. 29, pp. 155-173.

Audretsch, D. B., y Feldman, M. P. (1996): «R\&D Spillovers and the Geography of Innovation and Production», en The American Economic Review, 86, 3 , pp. 630-640.

Betran PÉREZ, C. (1995): Industria y crecimiento económico en el primer tercio del siglo XX, España, 1913-1929, tesis doctoral inédita.

- (1997): «Tamaño de mercado y crecimiento industrial en España durante el primer tercio del siglo xx», en Revista de Historia Industrial, núm. 11, pp. 119-148.

CATALÁN, J. (1990): «Capitales modestos y dinamismo industrial: Origenes del sistema de fábrica en los valles guipuzcoanos, 1841-1918», en J. NADAL, y A. Carreras (eds.): Pautas regionales de la industrialización española (siglos XIX $y X X)$, Barcelona, Ariel, pp. 125-155.

CARRERAS, Albert (1984): «La producción industriai española, 1841-1981: construcción de un índice anual», en Revista de Historia Económica, núm. 1, pp. 127-157.

- (1987): «La industria: atraso y modernización», en La economía española en el siglo XX. Una perspectiva histórica, Barcelona, Ariel, pp. 280-312.

- (1990): «España en las tipologías de la industrialización», en Industrialización española: estudios de bistoria cuantitativa, cap. 4, Madrid, Espasa Calpe, pp. 97-110.

DAVID, Paul (1985): «Clio and economics of QWERTY», en American Economic Review, núm. 75, may, pp. 332-337.

DAVid, Paul A., y Rosenbloom JoshuA, L. (1990): «Marshallian Factor externalities and the Dinamics of Industrial Location», en Journal of Urban Economics, núm. 28. pp. 349-370.

FAINI, R. (1984): «Increasing returns, nontraded inputs, and regional developments», Economic Journal, núm. 94, june, pp. 304-323.

GAROFOLI, G. (1993): «Economic development, organization of production and territory», en Revue d'Economie Industrielle, núm. (0) 64, 2. ${ }^{\circ}$ trimestre, pp. 22-37.

Gaudemar, J. P.; Gaffard, J. L., y Mougeot, M. (1993): «L'écomiste face à la localisation des activités», en Revue économique, núm. 4, pp. 645-651. 
Glaeser, E. L.; Kallal, H. D.; Scheinkman, J. A., y Schleifer, A. (1992): «Growth in Cities», en Joumal of Political Economy, 100, 6, pp. 1126-1152.

HeNDERSON, V. (1994): «Externalities and industrial development», NBER Working Paper, núm. 4730, may.

Herce, J. A.; De Lucio, J. J., y Goicolea, A. (1996): «La industria en las comunidades autónomas: 1978-1992», en Papeles de Economía, núm. 67, pp. 134-147.

HoOver, E. M. (1948): The Location of Economic Activity, New York, McGraw-Hill. Hotelling, H. (1929): «Stability in competition», Economic Joumal, núm. 39. IsARD, W. (1956): Location and Space-Economy, Cambridge, MA, The MIT Press.

KIM, Sukkoo (1995): «Expansion of markets and the geographic distribution of economic activities: the trends in US regional manufacturing structure, 1860-1987», en Quaterly Journal of Economics, núm. 110, pp. 881-908.

KrugmaN, Paul (1991a): «Increasing Returns and Economic Geography», Journal of Political Economy, vol. 99, núm. 31, pp. 183-499.

- (1991b): «History and Industry Location: The Case of Manufacturing Belt», American Economic Review, vol. 81, núm. 2, pp. 80-83.

- (1991c): «History versus expectations», en Quaterly Joumal of economics, núm. 106, may, pp. 651-667.

- (1991d): Geography and Trade, Cambridge MA, The MIT Press (trad. castellano). Geografia y comercio, Barcelona, A. Bosch.

Lösch, A. (1940): Die raumliche Ordnung der Urtsschaft, New Haven Yale University Press. Versión inglesa (1954, y 6." ed., 1973): The economics of Location, New Haven and London, Yale University Press.

Marshall, A. (1920): Principles of Economics, London, Macmillan.

Moses, L. N. (1958): «Location and the Theory of Production», Quaterly Joumal of Economics, núm. 72, pp. 259-272.

Murphy, K.; Shleifer, A., y VishnY, R. (1989): «Industrialization and the Big Push», en Journal of Political Economy, núm. 97 (october), pp. 1003-1026.

NADAL, J. (1987): «La industria fabril española en 1900», en J. NADAL; A. CARRERAS y C. SudriA (comps.): La economía española en el siglo XX, Barcelona, Ariel, pp. 23-61.

Nadal, J., y Tafunell, X. (1992): San Martí de Provencals, pulmó industrial de Barcelona (1847-1992), Barcelona, Columna Edicions, S. A.

Pyke, F.; BecatTini, G., y SENGERBERGER, W. (1990) (eds.): Industrial districts and inter-firm co-operation in Italy, Geneva, International Institute for labour studies.

Romer, P. (1986): «Increasing Returns and Long-Run Growth», en Journal of Political Economy, 94, 5, pp. 1002-37.

WEBER, A. (1909): Über den Standort der Industrien, University of Chicago Press, Chicago. Versión inglesa (1929): Theory of the location of industries, University of Chicago Press.

Young, Ruth C. (1986): «Industrial Location and Regional Change: The United States and New York State», Regional Studies, vol. 20, núm. 4, pp. 341-369. 